\title{
Vinblastine differs from Taxol as it inhibits the malignant phenotypes of NSCLC cells by increasing the phosphorylation of Op18/stathmin
}

\author{
FANG SHEN ${ }^{1,2}$, DAN LONG $^{1}$, TING YU ${ }^{1}$, XIAN CHEN $^{1}$, YING LIAO $^{1}$, YI WU $^{2}$ and XUECHI LIN ${ }^{1,2}$ \\ ${ }^{1}$ Department of Medical Laboratory, Changsha Medical University, Changsha, Hunan 410219; \\ ${ }^{2}$ Department of Clinical Laboratory, The First Affiliated Hospital of Hunan Normal University, \\ Changsha, Hunan 410000, P.R. China
}

Received August 15, 2016; Accepted October 10, 2016

DOI: $10.3892 /$ or.2017.5469

\begin{abstract}
Taxol (paclitaxel) and vinblastine (VBL) are both efficacious chemotherapeutic agents that target the microtubules of tumor cells, but each functions in a mutual antagonistic manner. Op18/stathmin is a small molecular phosphoprotein which promotes depolymerization of microtubules. Non-small cell lung cancer (NSCLC) NCI-H1299 cells were employed to compare the curative effects of VBL and Taxol and explore the correlation between drug sensitivity and Op18/stathmin signaling. The present study found that VBL obviously promoted cellular apoptosis and initiated activation of caspase 3 and 9, and inhibited cell proliferation and colony formation, as well as cell migration in the NCI-H1299 cells in contrast with Taxol. VBL did not affect the expression of Op18/stathmin, but increased its phosphorylation at all 4 serine sites. Conversely, Taxol mainly decreased the expression of Op18/stathmin and the phosphorylation at Ser25 and Ser63 sites. Silencing of Op18/stathmin by RNA interference (RNAi) led to a great reduction in the differences in the cell proliferation inhibition between VBL and Taxol. VBL treatment notably weakened the expression of PP $2 \mathrm{~A}, \mathrm{Bcl}-2, \mathrm{NF}-\kappa \mathrm{B}$ and interleukin-10 (IL-10) and autocrine IL-10 compared with Taxol; whereas PP2A was substantially increased following Taxol induction. High expression of Op18/stathmin was found to be negatively correlated with the sensitivity of Taxol in the NSCLC cells, but had a minor impact on VBL cytotoxicity.
\end{abstract}

Correspondence to: Professor Yi Wu, Department of Clinical Laboratory, The First Affiliated Hospital of Hunan Normal University, 61 Liberation West Road, Furong, Changsha, Hunan 410000, P.R. China

E-mail: wuyi19701210@sina.com

Professor Xuechi Lin, Department of Medical Laboratory, Changsha Medical University, $9 \mathrm{~km}$ stop at Leifeng Road, Wangcheng District, Changsha, Hunan 410219, P.R. China

E-mail:xuechilin71@126.com

Key words: vinblastine, Taxol, Op18/stathmin, signal regulation, non-small cell lung cancer
These findings revealed that both VBL and Taxol induce cell apoptosis through Op18/stathmin, but the mechanisms are completely different. VBL is an attractive alternative to the treatment of Taxol-resistant tumors with high expression of Op18/stathmin.

\section{Introduction}

Chemotherapeutic agents that target microtubules are mainly divided into two categories based on their functions. One agent, Taxol (paclitaxel), is a microtubule stabilizer which leads to obstacles in chromosome separation and the cell cycle process by promoting microtubule polymerization. Taxol is widely prescribed for the treatment of solid tumors including advanced ovarian and breast cancer, non-small cell lung cancer (NSCLC) and Kaposi's sarcoma $(1,2)$. The other is vinblastine (VBL), a microtubule destabilizer vinca alkaloid, which causes the dysfunction of cell proliferation by predominantly binding to $\alpha / \beta$ tubulin in order to disassemble microtubules. VBL is commonly applied to treat malignant lymphoma, osteosarcoma, breast, ovarian, gastric and lung cancer, Hodgkin's disease and choriocarcinoma $(3,4)$. Thereby, both Taxol and VBL interact with tubulin while in opposite patterns.

Op18/stathmin is a small molecular biomarker and is highly expressed in many carcinomas. Op18/stathmin integrates and relays intracellular and extracellular stimuli to regulate the equilibrium of microtubule dynamics through phosphorylated inactivation and dephosphorylated activation at 4 serine sites including Ser16, Ser25, Ser38 and Ser63, which is vital to the maintenance of malignant phenotypes in tumors (5-7). Our previous study confirmed that high expression of Op18/ stathmin was significantly correlated with Taxol resistance in 5 randomly selected human epithelial-derived carcinoma cell lines, including nasopharyngeal carcinoma CNE1, gastric MGC, breast cancer MCF-7, and hepatocellular carcinoma Hep3B-2 cells, as well as in NSCLC NCI-H1299 cells (8).

The present study aimed to compare the curative effects of VBL and Taxol in non-small cell lung cancer cells and to explore the correlation between drug sensitivity and Op18/stathmin signaling. 


\section{Materials and methods}

Cell culture. NSCLC NCI-H1299 and A549 cells were cultured in RPMI-1640 medium supplemented with $10 \%(\mathrm{v} / \mathrm{v})$ fetal bovine serum (FBS) and $1 \%$ penicillin/streptomycin and maintained at $37^{\circ} \mathrm{C}$ in $5 \% \mathrm{CO}_{2}$. Both cell lines were presented as a gift from Dr Yongguang Tao from the Cancer Research Institute of Central South University. NCI-H1299 was confirmed to be a Taxol-resistant cell line with high expression of Op18/stathmin in our previous study (8).

Antibodies and chemical reagents. Primary antibodies included rabbit polyclonal anti-stathmin (Calbiochem, La Jolla, CA, USA), rabbit monoclonal anti-stathmin (phospho-Ser16, -Ser25, -Ser38 and -Ser63) (Abcam, Cambridge, MA, USA), mouse monoclonal anti-caspase 8 , rabbit monoclonal anticaspase 9, rabbit monoclonal anti-PP2A C subunit (Cell Signaling Technology, Danvers, MA, USA), rabbit anti-phosphoserine (Invitrogen, Carlsbad, CA, USA), part antibodies including rabbit polyclonal anti-Bcl-2, rabbit polyclonal anti$\mathrm{NF}-\kappa \mathrm{B}$, mouse monoclonal anti $\beta$-actin, mouse monoclonal anti-caspase 3, mouse monoclonal anti-interleukin-10 (IL-10), which were purchased from Santa Cruz Biotechnology, Inc. (Santa Cruz, CA, USA).

Secondary antibodies included rabbit anti-mouse HRP-conjugated IgG and goat anti-rabbit HRP-conjugated IgG (Santa Cruz Biotechnology, Inc.).

Taxol (sc-201439) and VBL (sc-491749) were also obtained from Santa Cruz Biotechnology, Inc., and were dissolved in dimethyl sulfoxide (DMSO) at appropriate concentrations, and were stored at $-20^{\circ} \mathrm{C}$.

Assessments of cell apoptosis. Upon reaching $80 \%$ confluency, the cells were treated with Taxol or VBL $(100 \mathrm{nM})$ for $24 \mathrm{~h}$, and the solvent DMSO was used as control. Then, the treated cells were harvested and stained with $500 \mu$ l specific binding buffer containing $5 \mu \mathrm{l}$ Annexin V-FITC and $5 \mu \mathrm{l}$ propidium iodide for $10 \mathrm{~min}$ at room temperature in the dark. Analysis of cell apoptosis was performed by flow cytometry at the The Second Xiangya Hospital Affiliated with Central South University.

MTT assays. Cells (5,000/well) in a logarithmic growth phase were planted into 96-well plates and treated with Taxol or VBL $(100 \mathrm{nM})$. Next, $10 \mu \mathrm{l}$ of $5 \mathrm{mg} / \mathrm{ml}$ 3-(4,5-dimethylthiazol-yl)2,5-diphenyl tetrazolium bromide (MTT) was added to each well at time points of $0,24,48$ and $72 \mathrm{~h}$, and the cells were subsequently incubated at $37^{\circ} \mathrm{C}$ for $4 \mathrm{~h}$. After the culture supernatants were removed, $100 \mu 1$ DMSO was added for $10 \mathrm{~min}$, and the absorbance was measured at a $490-\mathrm{nm}$ wavelength. Six parallel wells were performed.

Cellular proliferative viability was calculated using the following formulation: Relative proliferative percentages $(\%)=\left(\mathrm{OD}_{\text {treatment }} / \mathrm{OD}_{\text {control }}\right) \times 100 \%$. The percentage of the DMSO control was designed as $100 \%$ at all time points.

Colony formation assays. Cells (2,000/well) were cultured in media with $10 \mathrm{nM}$ Taxol or VBL in 6-well plates for 1-2 weeks. The culture was suspended when obvious colonies were observed by the naked eye. Cells were fixed with methanol, and then stained with $0.1 \%$ crystal violet for $30 \mathrm{~min}$. Colonies with $>50$ cells/colony were counted using an inverted microscope. The experiment was carried out in triplicate independently. The relative colony formation efficiency $(\%)=($ colony number $/ 2,000) \times 100 \%$.

Wound healing assays. Cells were grown in 6-well plates to a monolayer. The cell monolayer was then wounded by creating a scratch using a $200-\mu 1$ pipette tip. Old medium was replaced with fresh medium containing $100 \mathrm{nM}$ Taxol or VBL. The status of wound healing was monitored at time points of 0 , 12,24 and $36 \mathrm{~h}$, and images were captured for assaying the capability of cell migration in a 2-dimensional plane.

Transwell assays. Capability of cell migration in a 3-dimensional space was assessed by Transwell assays according to the protocol described in a previous study (8). Approximately $4 \times 10^{4}$ cells were seeded in $200 \mu \mathrm{l}$ RPMI-1640 medium with $0.2 \%$ FBS in the upper chambers of a Transwell, while $800 \mu 1$ media with $10 \%$ FBS was placed in the lower chambers. Twenty-four hours later, the media were removed and the cells were wiped off from the upper chambers using a cotton swab. The remaining migrated cells on the back of the bottom membrane in the upper chamber were fixed with $100 \%$ methanol and stained with $0.1 \%$ crystal violet. Finally, the Transwell plates were mounted and observed under an inverted microscope, and the migrated cells were counted and images were captured.

Western blot analysis. Protein extracts were collected according to our previous studies $(9,10)$. Briefly, cells were lysed with lysis buffer (50 mM Tris-HCl pH 8.0, 1 mM EDTA, 2\% SDS, $5 \mathrm{mM}$ DTT, $10 \mathrm{mM}$ PMSF), and then denatured in boiling water for sonication. Protein concentrations were determined using BCA protein assay reagent (Pierce, Rockford, IL, USA).

Protein extracts $(50 \mu \mathrm{g})$ were separated by $10 \%$ SDS-PAGE, electrotransferred to nitrocellulose membrane, blocked with buffer containing 5\% non-fat milk and incubated with primary antibodies overnight at $4^{\circ} \mathrm{C}$ and HRP-conjugated secondary antibodies for $1 \mathrm{~h}$ at room temperature and developed with an enhanced chemiluminescence detection kit (Thermo Fisher Scientific, Waltham, MA, USA).

IP-western blot assays. Cells were lysed in immunoprecipitation (IP) lysis buffer (50 mM Tris- $\mathrm{HCl}, 150 \mathrm{mM} \mathrm{NaCl}, 10 \%$ NP-40, $1 \mathrm{mM}$ EDTA, $10 \%$ glycerol, $10 \mathrm{mM} \mathrm{NaF}, 1 \mathrm{mM}$ $\mathrm{Na}_{3} \mathrm{VO}_{4}, 1 \mathrm{mM}$ DTT, $1 \mathrm{mM}$ PMSF and protease inhibitor cocktail tablet), and then the supernatant was collected for detection of the total level of phospho-Op18/stathmin.

Magnetic immunobeads (cat\#: 10002D; Thermo Fisher Scientific) were used to bind the anti-stathmin antibody and pull-down Op18/stathmin for western blot analysis as previously described (9).

Autocrine IL-10 detection. Equal number of cells were planted in 6-well plates and cultured. The old medium was replaced with fresh medium without phenol red indicator in the presence of Taxol or VBL $(100 \mathrm{nM})$ when cells reached $80 \%$ confluency. Twenty-four hours later, the media were collected and centrifuged at 13,000 rpm for $30 \mathrm{~min}$. Autocrine IL-10 in the 
A
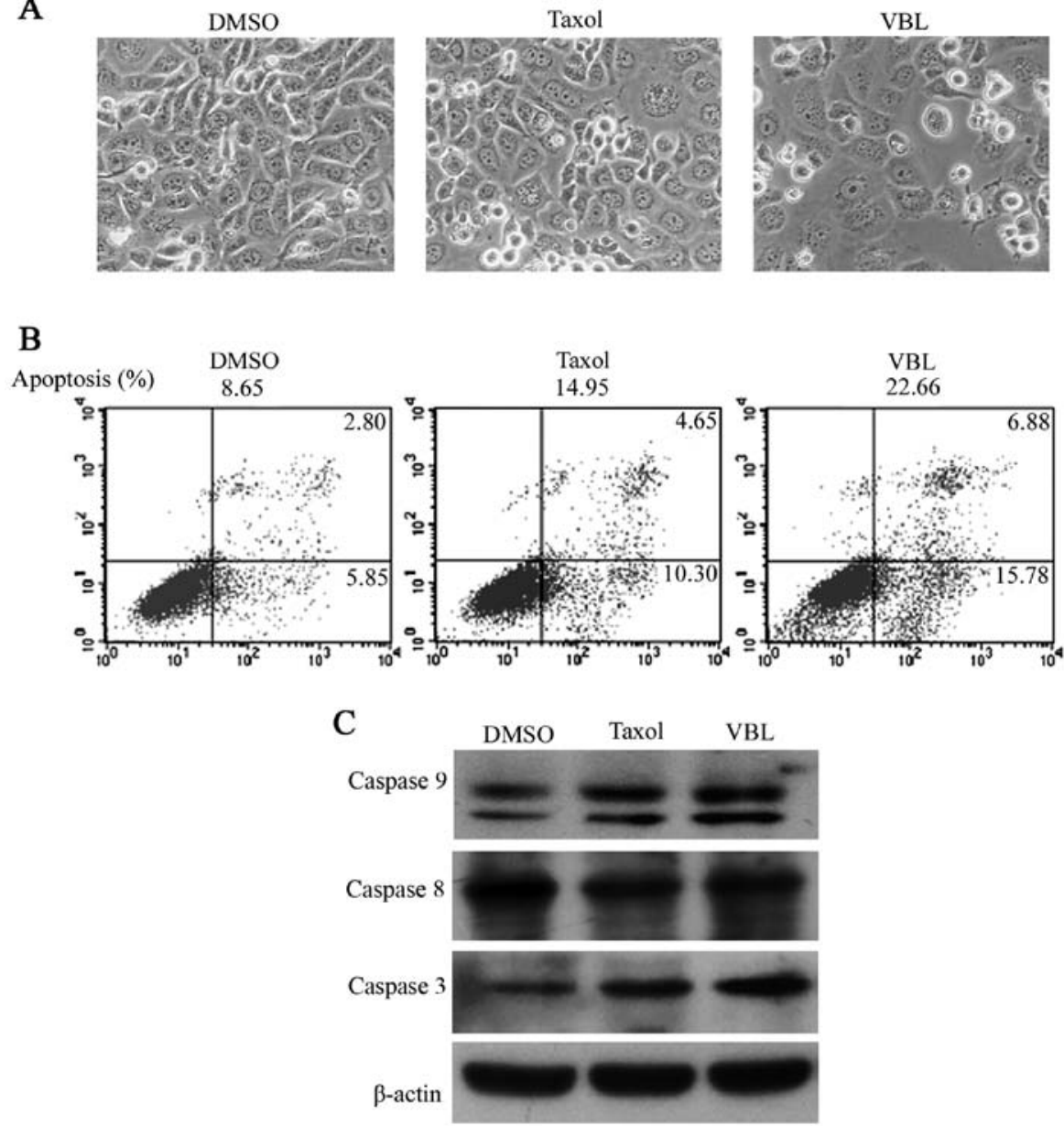

Figure 1. VBL induces a higher cell apoptotic ratio than Taxol. (A) Morphological images show the condition of cellular growth following treatment with DMSO, Taxol and VBL. (B) Analysis of cellular apoptosis by FCM. (C) Western blotting showing the expression of caspase 3,8 and 9 in the three treatment groups. VBL, vinblastine; Taxol, paclitaxel.

separated supernatant was detected by ELISA using the human IL-10 ELISA kit (Cusabio, Wuhan, China). All experiments were carried out in triplicate.

Construction of the RNA interference plasmid targeting Op18/stathmin. Plasmids pGCsilencer-U6/Neo/GFP-RNAi (RNAi) with the RNAi gene targeting the coding region of Op18/stathmin at 5'-AGAGAAACTGACCCACAAA-3' (GenBank no. 53305, sequence 374-393) and pGC-silencer-U6/ Neo/GFP-non (Non) with an inserted non-coding sequence and pGC-silencer-U6/Neo/GFP (Blank) without any fragments inserted, were constructed at an early stage and were introduced into cells by Lipofectamine 2000 (Invitrogen) as described in our previous studies $(8,10)$.

Statistical analysis. All statistical calculations were performed using the statistical software program SPSS 17.0. Differences with $p$-values $<0.05$ were considered statistically significant.

\section{Results}

NCI-H1299 cells are more sensitive to VBL than to Taxol. Morphological images showed that cells grew to nearly complete confluency in the control DMSO, whereas cell distribution became gradually dispersed with the appearance of a few translucent floating cells in the Taxol treatment cell group. A large number of suspended cells emerged and only a minority of cells adhered to the wall at a low density in the VBL treatment group (Fig. 1A).

Cell apoptotic ratios were $8.65,14.95$ and $22.66 \%$ following treatment with the solvent (DMSO), Taxol and VBL, respectively, in the NCI-H1299 cells as detected by FCM. The apoptotic ratio was significantly higher for VBL treatment compared with Taxol (Fig. 1B).

Western blot analysis showed that both Taxol and VBL increased the expression levels of caspase 3 and 9, but to a greater extent following VBL treatment, while no obvious changes in caspase 8 were observed in the three treatment groups (Fig. 1C).

VBL significantly inhibits cell proliferation and colony formation compared with Taxol. By contrast with DMSO, the relative cell proliferation percentages were $84.24,55.11$ and $32.00 \%$, respectively, for VBL treatment at time points of 24, 48 and $72 \mathrm{~h}$, while these percentages were $95.86,91.47$ and $86.46 \%$, respectively, following Taxol treatment. The representative curve steeply declined with increasing exposure time to VBL, whereas, it declined slowly in the presence of Taxol (Fig. 2A). 

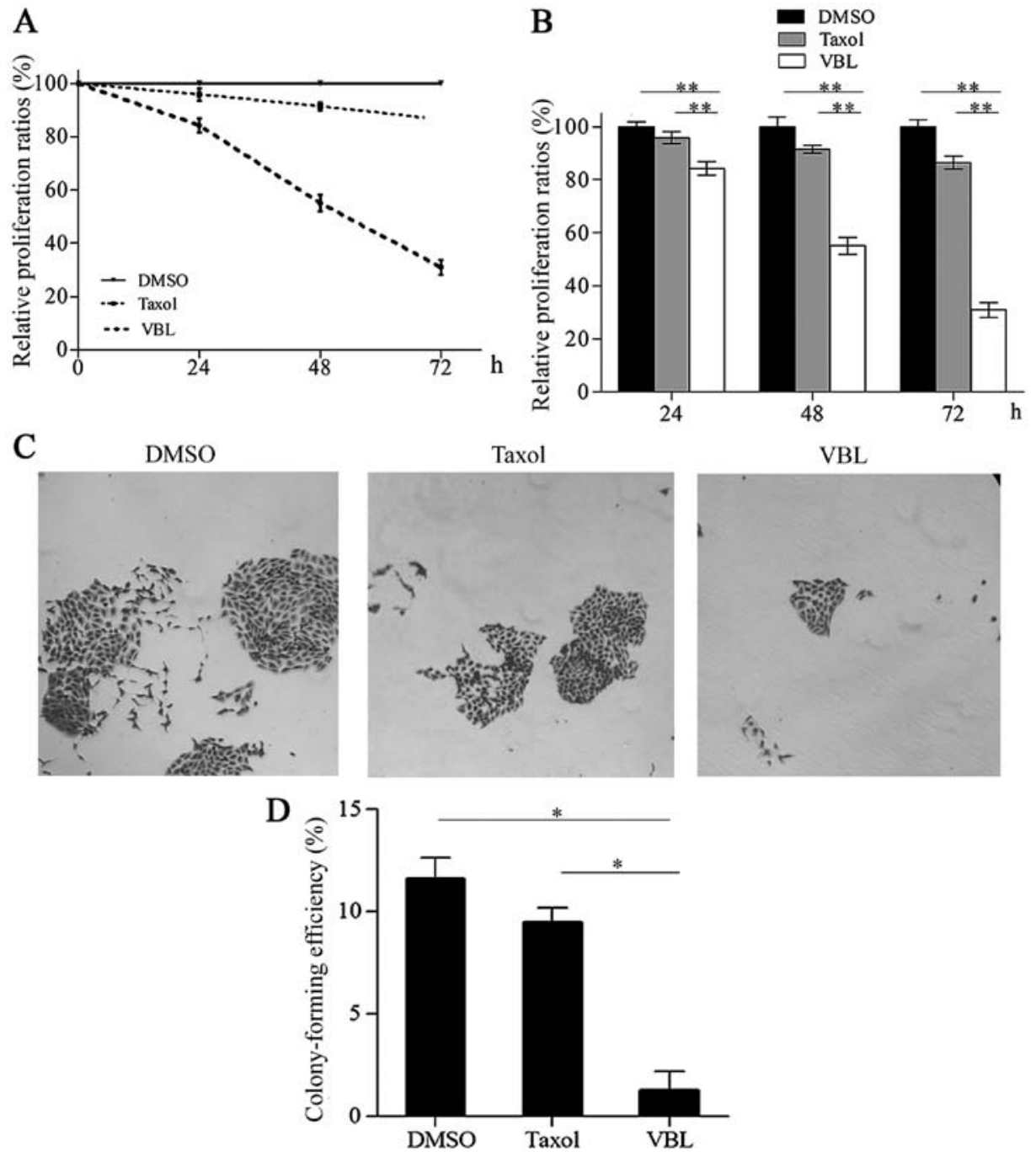

Figure 2. VBL significantly inhibits cell proliferation and colony formation compared with Taxol. (A) Curves represent relative proliferative trends of NCIH1299 cells in terms of the different treatments. (B) Histograms illustrate statistical differences in cellular proliferation between the VBL treatment group and the other two treatment groups. (C) Images show the status of colony formation for the different treatment groups. (D) VBL significantly inhibited colony formation compared with Taxol; ${ }^{*} \mathrm{p}<0.05,{ }^{* *} \mathrm{p}<0.01$. VBL, vinblastine; Taxol, paclitaxel.

Statistical differences existed in the proliferation percentages between VBL and that of the other two groups for Taxol and DMSO at 24, 48 and $72 \mathrm{~h}(\mathrm{p}<0.01)$ (Fig. 2B).

Colony formation assays revealed that only a few small colonies appeared in the VBL treatment group, while some relative larger colonies emerged in the Taxol treatment group. There were a large number of large colonies which generally merged into an extensive one with unclear borders in the control DMSO group (Fig. 2C). The mean colony formation efficiency was $11.60,9.47$ and $1.07 \%$, respectively, in the DMSO, Taxol and VBL treatment group. VBL significantly reduced the percentage of colony formation in comparison with the Taxol and DMSO treatment groups ( $<<0.05)$ (Fig. 2D).

VBL notably suppresses cell migration in contrast with Taxol. The scratch-wound trails obviously became narrow with time in the three groups, and were completely recovered at time point of $36 \mathrm{~h}$ in the DMSO treatment group, while the scratched areas nearly healed for Taxol induction. However, a large area of blank region still remained in the cells treated with VBL (Fig. 3A).
Transwell assay analysis revealed that the mean number of invading cells was 27,16 and 8, respectively, in the DMSO, Taxol and VBL treatment group. The number of migrated cells was greatly decreased following treatment with VBL when compared to the Taxol treatment group, whereas Taxol also inhibited cell migration in contrast with DMSO (Fig. 3B). Differences in the number of migrating cells were significant between the VBL group and the other two treatment groups; VBL was the most notable in inhibiting cell migration of a 3-dimensional space $(\mathrm{p}<0.01)$ (Fig. 3C).

$V B L$ promotes the phosphorylation of Op18/stathmin, while Taxol decreases the expression and phosphorylation of Op18/ stathmine. Western blot analysis revealed that the expression of Op18/stathmin was decreased by Taxol, but was not obviously affected by VBL (Fig. 4A). IP-western blotting showed that Taxol decreased the phosphorylation of Op18/stathmin; however, VBL upregulated the total level of phosphorylated Op18/stathmin (Fig. 4B).

VBL universally increased the level of phosphorylated Op18/stathmin at all 4 serine sites including Ser63, Ser38, 

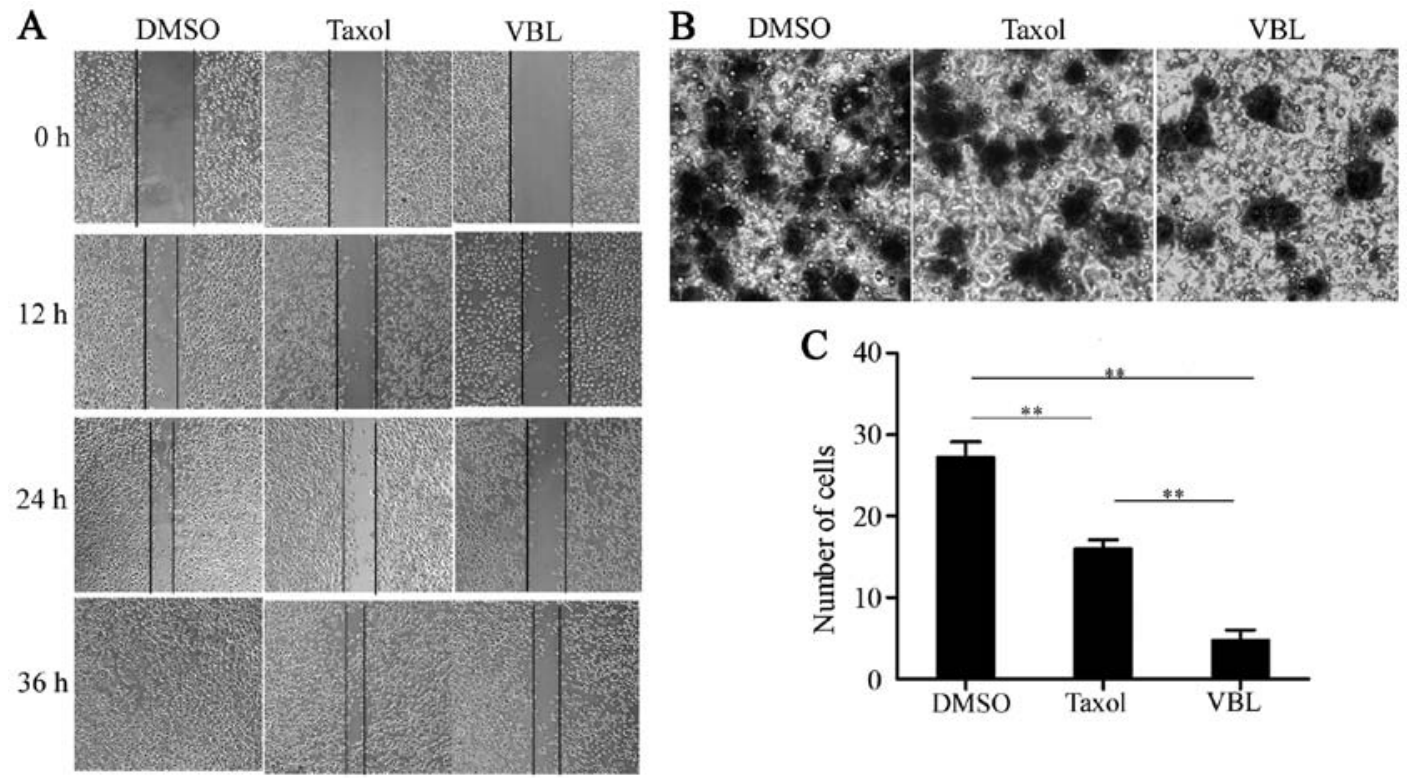

Figure 3. VBL notably inhibits cell migration in contrast with Taxol. (A) Wound healing assays demonstrated the status of cell migration in a 2-dimensional plane. (B) Transwell assay analysis shows the cell aggression in 3 dimensional space. (C) Histograms show the statistical differences in migrated cells in the different treatment groups; ${ }^{* *} \mathrm{p}<0.01$. VBL, vinblastine; Taxol, paclitaxel.

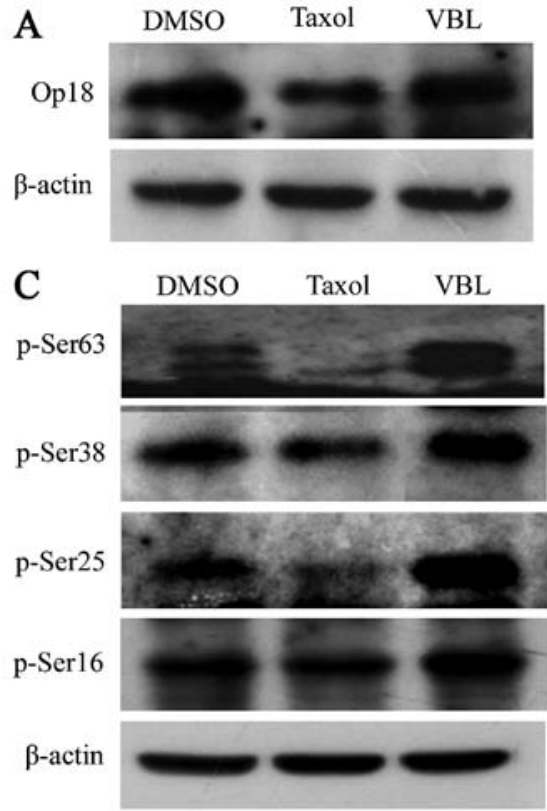

Ser25 and Ser16, while Taxol mainly reduced phosphorylated Op18/stathmin at Ser25 and Ser63 sites, particularly, p-Ser63Op18/stathmin was almost completely inhibited, yet Taxol did not influence the phosphorylation of Op18/stathmin at Ser16 and Ser38 (Fig. 4C).

Op18/stathmin RNAi reduces the difference in cell proliferation inhibition between VBL and Taxol. MTT analysis demonstrated that the proliferation ratios were 99.7,96.79 and $94.48 \%$, and $68.73,54.38$ and $50.88 \%$, at time points 24,48 and $72 \mathrm{~h}$ in the non-silenced and the RNAi groups respectively in comparison with the DMSO group. The representative curve of the RNAi group declined rapidly, the curve of the
B IP: Op18/stathmin pull down

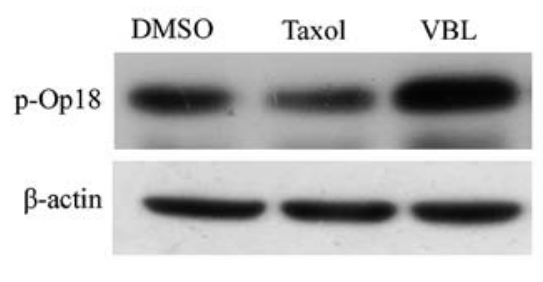

Figure 4. VBL increases the level of phosphorylated Op18/stathmin, while Taxol decreases the expression and phosphorylation of Op18/stathmin. (A) Western blotting shows the expression of Op18/stathmin. (B) IP-western blotting shows the total level of phospho(p)-Op18/stathmin. (C) VBL promotes the phosphorylation of Op18/stathmin at all 4 serine sites, while Taxol mainly decreases p-Op18/stathmin at Ser25 and Ser63. VBL, vinblastine; Taxol, paclitaxel. non-silenced group nearly overlapped the one of the Blank. After co-treatment with Op18/stathmin RNAi and VBL or Taxol, the relative proliferative ratios were $60.95,40.32$ and $27.16 \%$, and $58.69,33.20$ and $24.66 \%$, respectively, at the three time points, while the corresponding curves were highly similar between VBL and Taxol, which declined steeply compared with the one for single RNAi (Fig. 5A). Histograms showed that the difference was not significant between the Blank and the non-silenced group, but was increased between the RNAi groups and the blank control $(\mathrm{p}<0.01)$. The combination of RNAi and VBL or Taxol significantly inhibited cell proliferation in contrast with RNAi alone treatment $(\mathrm{p}<0.01)$, whereas Op18/stathmin RNAi reduced the difference in cell 

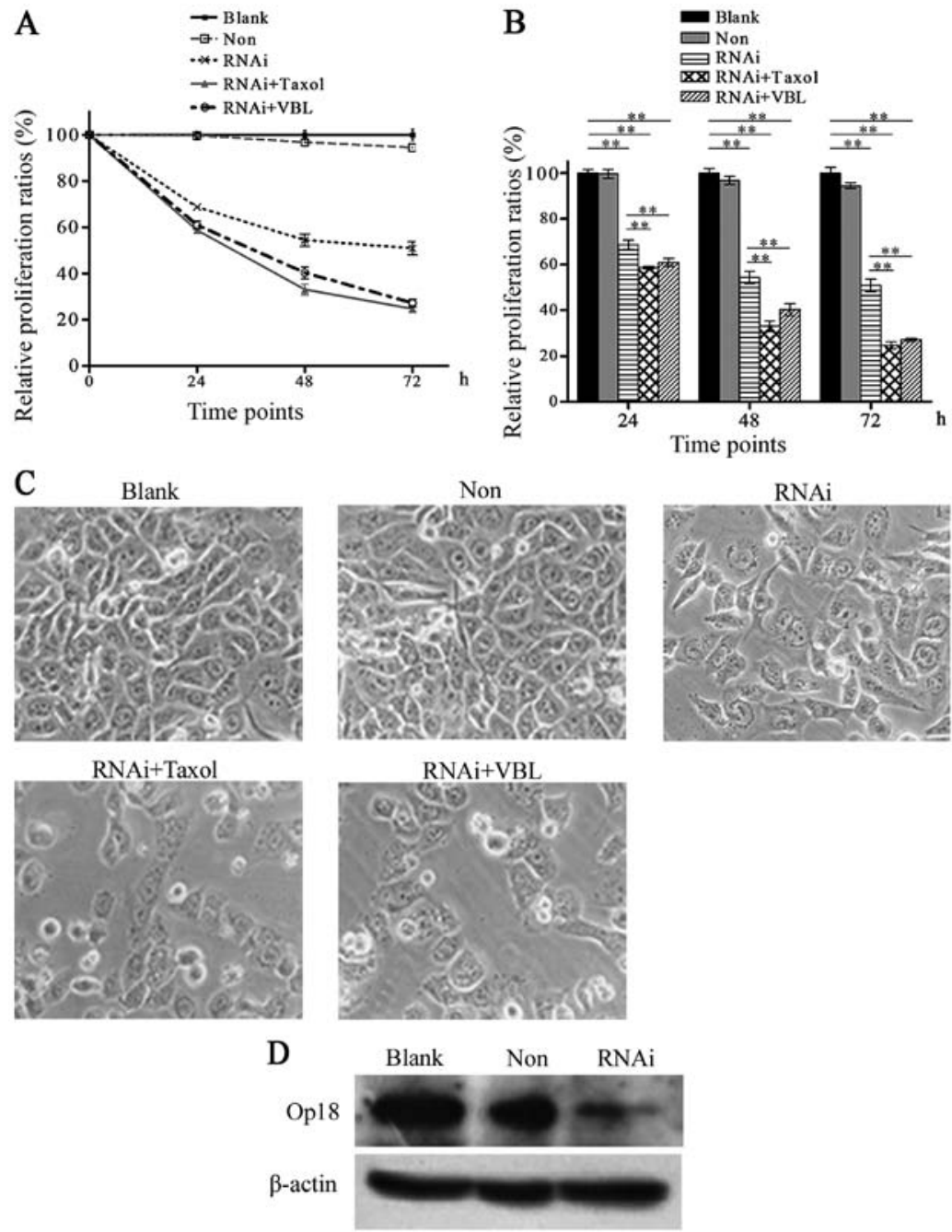

Figure 5. Op18/stathmin RNAi reduces the difference in cell proliferation inhibition between VBL and Taxol. (A) Curves represent the trends of cell proliferation for the different treatment groups. (B) Statistical differences are illustrated. (C) The conditons of cell growth are captured by images. (D) Op18/stathmin RNAi effectively inhibited the expression of Op18/stathmin; ** $<<0.01$. VBL, vinblastine; Taxol, paclitaxel.

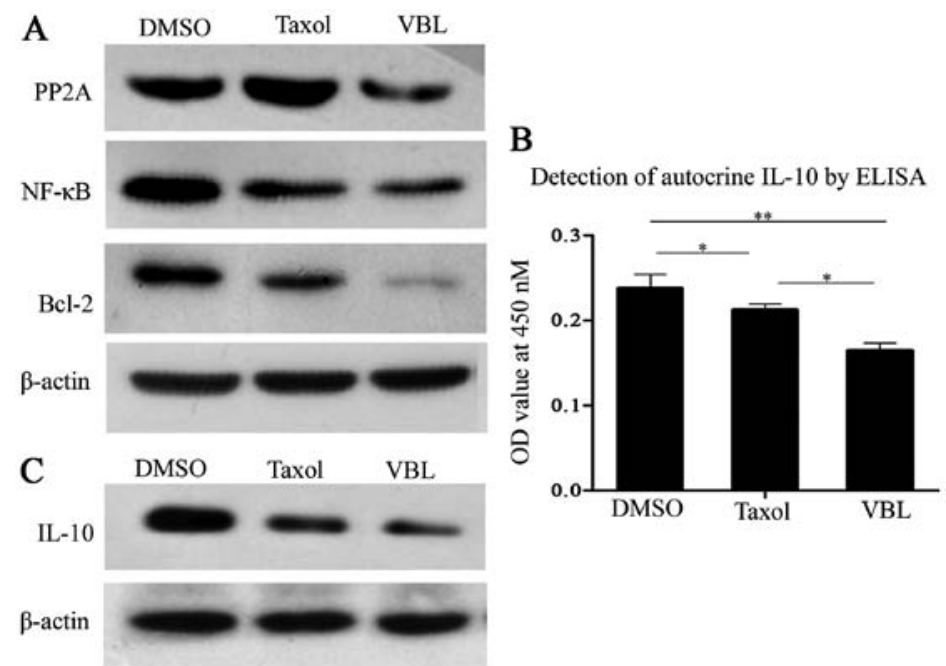

Figure 6. VBL decreases the expression of PP2A, Bcl-2, NF- $\mathrm{BB}$ and IL-10 and autocrine IL-10 in comparison with Taxol. (A) Western blotting shows the expression levels of PP2A, NF-kB and Bcl-2. (B) Detection of autocrine IL-10 from the culture supernatant by ELISA. (C) Western blot analysis shows the expression of IL-10 in the DMSO, Taxol and VBL treatment groups; ${ }^{*} \mathrm{p}<0.05,{ }^{* *} \mathrm{p}<0.01$. VBL, vinblastine; Taxol, paclitaxel.

proliferation inhibition between VBL and Taxol at all time points (Fig. 5B).
Cell growth images show that cell proliferation was obviously inhibited by RNAi, while the inhibition was increased 

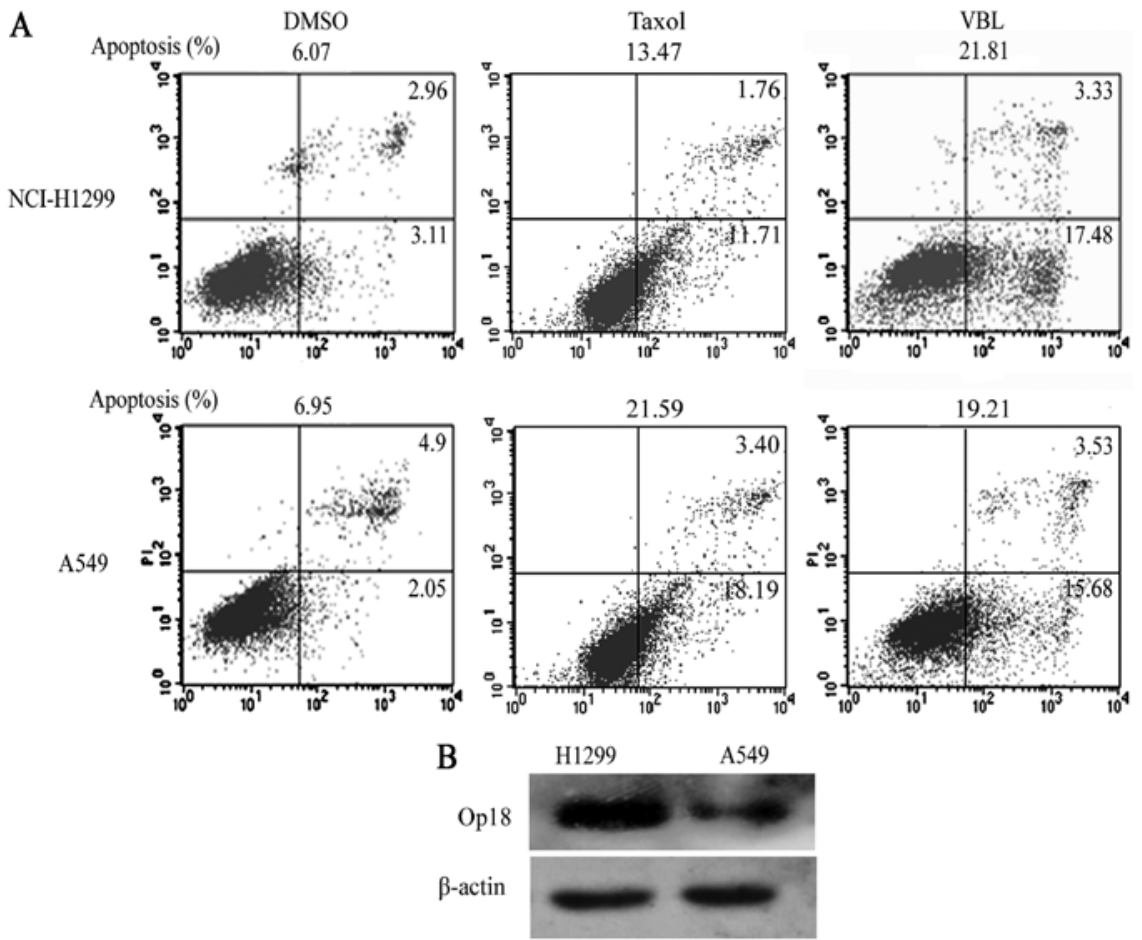

Figure 7. High expression of Op18/stathmin is negatively relevant to the sensitivity of Taxol in different NSCLC cells, but only exerted few impacts on VBL cytotoxicity. (A) FCM shows cellular apoptotic ratios in NCI-H1299 and A549 cells. (B) Western blotting shows the expression of Op18/stathmin. VBL, vinblastine; Taxol, paclitaxel.

following co-treatment of RNAi and VBL or Taxol. Cells became much sparser in the co-treated groups with RNAi than the RNAi alone treatment group (Fig. 5C).

Western blot analysis showed that Op18/stathmin RNAi effectively inhibited the expression of Op18/stathmin in the NCI-H1299 cells (Fig. 5D).

$V B L$ markedly weakens the expression levels of $P P 2 A$, $B c l-2, N F-\kappa B$ and $I L-10$ and autocrine IL-10 in comparison with Taxol. Western blot analysis showed that Taxol notably enhanced the expression of protein phosphatase 2A (PP2A), but PP2A was substantially decreased by VBL, while both Taxol and VBL treatments decreased the expression of nuclear factor- $\kappa \mathrm{B}(\mathrm{NF}-\kappa \mathrm{B})$, B-cell lymphoma-2 (Bcl-2). However, the inhibitory effects were more notable for VBL induction than Taxol (Fig. 6A).

ELISA demonstrated that autocrine IL-10 from cell culture supernatants was decreased by Taxol and VBL, but VBL induced more obvious inhibitory effects when compared with Taxol. Histograms showed that the differences were significant between the VBL and the other two treatment groups (Fig. 6B).

Western blot analysis showed that both Taxol and VBL inhibited the expression of IL-10 in the NCI-H1299 cells, but VBL was more effective than Taxol (Fig. 6C).

High expression of Op18/stathmin is negatively correlated with the sensitivity of Taxol in the different NSCLC cells, but only exerts a mild impact on VBL. FACS showed that cellular apoptotic percentages were $6.07,13.47$ and $21.81 \%$, respectively, in the NCI-H1299 cells in the DMSO, Taxol and VBL treatment groups, while they were $6.95,21.59$ and $19.21 \%$ respectively in A549 cells in the corresponding groups. NCI-H1299 cells presented notable resistance to Taxol when compared with VBL, but the sensitivity of Taxol was similar to VBL in the A549 cells (Fig. 7A).

Western blot analysis demonstrated that NCI-H1299 cells highly expressed Op18/stathmin compared with the A549 cells (Fig. 7B).

\section{Discussion}

Op18/stathmin is thought to be closely associated with cellular proliferation, differentiation and drug resistance of tumors (11-14). Our previous studies demonstrated that Op18/stathmin is regulated by multiple kinases including cyclin dependent 2 (CDC2) and extracellular signal regulated kinase (ERK), and that high expression of Op18/stathmin is positively correlated with the resistance of Taxol in different epithelial-derived carcinoma cells (8-10).

The present study demonstrated that NCI-H1299 cells were more sensitive to VBL than to Taxol. VBL obviously promoted cell apoptosis through initiating the activation of caspase 3 and 9, and inhibited cell proliferation, colony formation and cell migration in contrast with Taxol. VBL mainly decreased the activity of Op18/stathmin by triggering the phosphorylation of Op18/stathmin at all 4 serine sites. On the contrary, Taxol decreased the expression of Op18/stathmin and the phosphorylation at Ser25 and Ser63. In short, VBL, completely different from Taxol, mediates the Op18/stathmin signaling pathway by increasing the phosphorylation of Op18/ stathmin. Other studies also confirmed that the phosphorylation of Op18/stathmin at 4 serine residues is involved in cell apoptosis induced by exogenous tumor necrosis factor (TNF) in mouse fibrosarcoma L929 cells, and wee-1 knockdown 
promoted the sensitivity to VBL through augmenting phosphorylation of Op18/stathmin at Ser25 and Ser63 in human breast carcinoma BT20ST1 cells. Blocking ERK by PD98059 enhanced the sensitivity to Taxol through decreasing the expression of Op18/stathmin and the phosphorylation in NCI-H1299 cells $(9,15,16)$.

Previous studies demonstrated that silencing of Op18/ stathmin by RNAi promoted the sensitivity of breast cancer BT549 cells to Taxol and VBL as well as the sensitivity of nasopharyngeal carcinoma CNE1 cells to Taxol and high grade differentiated CNE1 xenografted tumors in nude mice $(16,17)$. The present study found that Op18/stathmin RNAi reduced the differences in cellular proliferation inhibition between VBL and Taxol, which implies that Op18/stathmin signaling mediates the sensitivities of both VBL and Taxol.

PP2A is an important serine/threonine phosphatase acting to dephosphorylate Op18/stathmin (18). NF- $\kappa \mathrm{B}$ is a transcription factor involved in cell survival and proliferation, which interacts with Op18/stathmin to promote tumor growth and predicts poor prognosis of pancreatic cancer (19-21). IL-10 is a pleiotropic cytokine which plays a vital role in tumor growth and drug resistance, of which high expression is associated with poor prognosis in tumor patients (22-24). $\mathrm{Bcl}-2$ is a typical protein against cellular apoptosis induced by chemotherapeutics $(25,26)$. The present study showed that the expression of PP2A was increased by Taxol, but decreased by VBL, which coincided with the status of phospho-Op18/ stathmin for Taxol or VBL induction, while both Taxol and VBL inhibited the expression levels of NF- $\mathrm{KB}, \mathrm{Bcl}-2$ and IL-10 as well as autocrine IL-10 in NCI-H1299 cells, but the inhibitory effects were more obvious for VBL than for Taxol.

It has been reported that overexpression of Op18/stathmin markedly decreases microtubule binding with Taxol and increased binding with VBL, downregulated the sensitivity to Taxol, but to VBL to a lesser extent in human breast cancer cells $(27,28)$. Others have found that high expression of Op18/ stathmin increased the sensitivity to vindesine and vincristine (one of vinca alkaloids) in human lung carcinoma cells, while crystallographic analysis showed that Op18/stathmin increased VBL binding to tubulin. Conversely, VBL also increased Op18/stathmin binding to tubulin in vitro (29-31). The present study indicated that high expression of Op18/ stathmin was negatively associated with the sensitivity of Taxol in different NSCLC cell lines, but exerted slight impact on VBL cytotoxity, which is an attractive alternative for the treatment of Taxol-resistant tumors with highly expressed Op18/stathmin.

\section{Acknowledgements}

The present study was funded by the National Natural Science Foundation of China (grant no. 81272274), the Key Project of Hunan Province Scientific Research of Colleges and Universities (no. 12A018), and the Natural Science Foundation of Hunan Province (no. 12JJ3104).

\section{References}

1. Hennessy BT, Coleman RL and Markman M: Ovarian cancer. Lancet 374: 1371-1382, 2009.
2. Takashima S, Kiyoto S, Takahashi M, Hara F, Aogi K, Ohsumi S, Mukai R and Fujita Y: Clinical experience with nanoparticle albumin-bound paclitaxel, a novel taxane anticancer agent, and management of adverse events in females with breast cancer. Oncol Lett 9: 1822-1826, 2015.

3. Coderch C, Morreale A and Gago F: Tubulin-based structureaffinity relationships for antimitotic Vinca alkaloids. Anticancer Agents Med Chem 12: 219-225, 2012.

4. Koontz MZ, Horning SJ, Balise R, Greenberg PL, Rosenberg SA, Hoppe RT and Advani RH: Risk of therapy-related secondary leukemia in Hodgkin lymphoma: The Stanford University experience over three generations of clinical trials. J Clin Oncol 31: 592-598, 2013.

5. Lin XC and Cao Y: Advances in the regulation of the rignals on Op18/stathmin. Life Science Res 11: 195-999, 2007.

6. Lin XC, Liao Y, Yang J, Su L, Zou H, Zuo Y and Zou L: Regulation of the drug resistance of carcinoma cells mediated by Op18/stathmin. Chem Life 33: 265-268, 2013.

7. Nemunaitis J: Stathmin 1: A protein with many tasks. New biomarker and potential target in cancer. Expert Opin Ther Targets 16: 631-634, 2012.

8. Lin X, Liao Y, Xie J, Liu S, Su L and Zou H: Op18/stathmin is involved in the resistance of Taxol among different epithelial carcinoma cell lines. Cancer Biother Radiopharm 29: 376-386, 2014.

9. Lin X, Liao Y, Chen X, Long D, Yu T and Shen F: Regulation of oncoprotein $18 /$ stathmin signaling by ERK concerns the resistance to Taxol in non-small cell lung cancer cells. Cancer Biother Radiopharm 31: 37-43, 2016

10. Lin X, Tang M, Tao Y, Li L, Liu S, Guo L, Li Z, Ma X, Xu J and Cao Y: Epstein-Barr virus-encoded LMP1 triggers regulation of the ERK-mediated Op18/stathmin signaling pathway in association with cell cycle. Cancer Sci 103: 993-999, 2012

11. Gupta KK, Li C, Duan A, Alberico EO, Kim OV, Alber MS and Goodson HV: Mechanism for the catastrophe-promoting activity of the microtubule destabilizer Op18/stathmin. Proc Natl Acad Sci USA 110: 20449-20454, 2013.

12. Hsu HP, Li CF, Lee SW, Wu WR, Chen TJ, Chang KY, Liang SS, Tsai CJ and Shiue YL: Overexpression of stathmin 1 confers an independent prognostic indicator in nasopharyngeal carcinoma. Tumour Biol 35: 2619-2629, 2014.

13. Trovik J, Wik E, Stefansson IM, Marcickiewicz J, Tingulstad S, Staff AC, Njolstad TS, Vandenput I, Amant F, Akslen LA, et al; MoMaTec Study Group: Stathmin overexpression identifies high-risk patients and lymph node metastasis in endometrial cancer. Clin Cancer Res 17: 3368-3377, 2011.

14. Werner HM, Trovik J, Halle MK, Wik E, Akslen LA, Birkeland E, Bredholt T, Tangen IL, Krakstad C and Salvesen HB: Stathmin protein level, a potential predictive marker for taxane treatment response in endometrial cancer. PLoS One 9: e90141, 2014.

15. Vancompernolie K, Boonefaes T, Mann M, Fiers W and Grooten J: Tumor necrosis factor-induced microtubule stabilization mediated by hyperphosphorylated oncoprotein 18 promotes cell death. J Biochem 275: 3876-3882, 2000.

16. Alli E, Yang JM, Ford JM and Hait WN: Reversal of stathminmediated resistance to paclitaxel and vinblastine in human breast carcinoma cells. Mol Pharmacol 71: 1233-1240, 2007.

17. Lin X, Yu T, Zhang LX, Chen SY, Chen X, Liao Y, Dan L and Shen F: Silencing Op18/stathmin by RNA interference promotes the sensitivity of nasopharyngeal carcinoma cells to taxol and high grade differentiation of xenografted tumours in nude mice. Basic Clin Pharmacol, 2016.

18. Tournebize R, Andersen SSL, Verde F, Dorée M, Karsenti E and Hyman AA: Distinct roles of PP1 and PP2A-like phosphatases in control of microtubule dynamics during mitosis. EMBO J 16: 5537-5549, 1997.

19. Hayden MS and Ghosh S: NF- $\kappa B$, the first quarter-century: Remarkable progress and outstanding questions. Genes Dev 26: 203-234, 2012.

20. Hoesel B and Schmid JA: The complexity of NF- $\mathrm{B}$ signaling in inflammation and cancer. Mol Cancer 12: 86, 2013.

21. Lu Y, Liu C, Cheng H, Xu Y, Jiang J, Xu J, Long J, Liu L and $\mathrm{Yu} \mathrm{X}$ : Stathmin, interacting with $\mathrm{Nf}-\kappa \mathrm{B}$, promotes tumor growth and predicts poor prognosis of pancreatic cancer. Curr Mol Med 14: 328-339, 2014.

22. Itakura E, Huang RR, Wen DR, Paul E, Wünsch PH and Cochran AJ: IL-10 expression by primary tumor cells correlates with melanoma progression from radial to vertical growth phase and development of metastatic competence. Mod Pathol 24: 801-809, 2011. 
23. Mahipal A, Terai M, Berd D, Chervoneva I, Patel K, Mastrangelo MJ and Sato T: Tumor-derived interleukin-10 as a prognostic factor in stage III patients undergoing adjuvant treatment with an autologous melanoma cell vaccine. Cancer Immunol Immunother 60: 1039-1045, 2011

24. Danoch H, Kalechman Y, Albeck M, Longo DL and Sredni B: Sensitizing B- and T- cell lymphoma cells to paclitaxel/abraxane-induced death by AS101 via inhibition of the VLA-4-IL10-survivin axis. Mol Cancer Res 13: 411-422, 2015.

25. Moldoveanu T, Follis AV, Kriwacki RW and Green DR: Many players in BCL-2 family affairs. Trends Biochem Sci 39: 101-111, 2014.

26. Delbridge ARD, Grabow S, Strasser A and Vaux DL: Thirty years of BCL-2: Translating cell death discoveries into novel cancer therapies. Nat Rev Cancer 16: 99-109, 2016.

27. Alli E, Bash-Babula J, Yang JM and Hait WN: Effect of stathmin on the sensitivity to antimicrotubule drugs in human breast cancer. Cancer Res 62: 6864-6869, 2002.
28. Purani I, Tejada A and Mistry SJ: Effects of combination of microtubule depolymerizing peptides and vinblastine in breast cancer. Cancer Res 73 (Suppl 8): S3275-S3275, 2013.

29. Nishio K, Nakamura T, Koh Y, Kanzawa F, Tamura T and Saijo N: Oncoprotein 18 overexpression increases the sensitivity to vindesine in the human lung carcinoma cells. Cancer 91: 1494-1499, 2001.

30. Devred F, Tsvetkov PO, Barbier P, Allegro D, Horwitz SB, Makarov AA and Peyrot V: Stathmin/Op18 is a novel mediator of vinblastine activity. FEBS Lett 582: 2484-2488, 2008.

31. Malesinski S, Tsvetkov PO, Kruczynski A, Peyrot V and DevredF: Stathmin potentiates vinflunine and inhibits Paclitaxel activity. PLoS One 10: e0128704, 2015. 